\title{
La forma de tratamiento nominal huevón en Chile: análisis empírico de conversaciones cotidianas informales
}

\author{
The nominal form of address huevón in Chile: \\ Empirical analysis of informal daily conversations
}

\section{Kris Helincks \\ Ghent University}

\begin{abstract}
Este artículo examina el uso de la forma de tratamiento chilena huevón en interacciones cotidianas. Huevón es uno de los vocativos más particulares del habla informal de Chile y se destaca tanto por su alta frecuencia como por la riqueza del campo derivacional del que forma parte. Además, la forma puede expresar significados tanto positivos como negativos. Basado en un amplio corpus de interacciones informales espontáneas, se analiza la influencia de parámetros lingüísticos (fonéticos, sintácticos, semánticos, pragmáticos) y extra-lingüísticos (sociales, relacionales, situacionales) en el uso de huevón y su valoración en relación con la cortesía. Los resultados indican que todas las generaciones y principalmente los hombres lo utilizan, en los ámbitos diarios más informales, sobre todo entre íntimos y en roles igualitarios. Contrariamente a lo que sugieren su semántica original y su estigma de rasgo maleducado, su uso raras veces pretende o efectúa descortesía.
\end{abstract}

Palabras clave: vocativos, marcadores del discurso, español chileno, sociopragmática

\begin{abstract}
This article examines the use of the Chilean form of address huevón in daily interactions. Huevón is one of the most particular vocatives of informal speech in Chile and it stands out both for its high frequency as for the rich derivational field it is part of. Moreover, the form can express both positive and negative meanings. Based on a broad corpus of spontaneous informal interactions, the influence of linguistic (phonetic, syntactic, semantic, pragmatic) and extra-linguistic (social, relational, situational) parameters is analyzed on the use of huevón and its valorization in relation to politeness. The results indicate that all generations and principally men use it, in the most informal daily situations, mostly between intimates and in equalitarian roles.
\end{abstract}


Contrary to its original semantics and its stigma of uneducatedness, its use scarcely intends or effectuates impoliteness.

Keywords: vocatives, discourse markers, Chilean Spanish, sociopragmatics

\section{Introducción}

Este artículo discute el uso de la forma de tratamiento (FdT) nominal huevón ${ }^{1}$ en la lengua oral informal en Chile. Huevón es uno de los vocativos más particulares del chileno. Primero, su uso es muy común en el lenguaje informal. Poblete y Samaniego (1999: 184), por ejemplo, seleccionan huevón como una de las cien lexías propias de la "identidad nacional chilena". En su estudio de palabras tabúes, Hernes (2011: 36) comprueba que huevón es la palabra tabú más frecuente del corpus juvenil COLAs ${ }^{2}$. Además, es parte de una red de derivaciones singular y productiva a partir del núcleo "hueva". Entre las derivaciones más comunes se destacan (a) los sustantivos huevón, huevona y huevá(da), (b) el verbo huevear, (c) los adjetivos huevón/-a y ahuevonado/-a y (d) el marcador del discurso (MDD) huevón.

Originalmente, huevón expresa un insulto dirigido solo a hombres. Tradicionalmente se ha descrito huevón como un término estilístico "bajo" y "vulgar", típico de la clase más popular (Lenz, 1905, en Sáez-Godoy, 1984: 147). Sin embargo, actualmente la palabra implica una variedad de significados que se extienden entre el polo muy negativo como un insulto o palabra tabú y el polo más positivo como un MDD que mantiene y refuerza los lazos sociales entre interlocutores (Cortés, 2009: 252). También estilísticamente "se ha ido desplazando desde la lengua popular hacia la lengua común" (Sáez-Godoy, 1983: 147), prevaleciendo en situaciones informales y de mucha confianza entre interlocutores (Rojas, 2012: 157). Un fenómeno similar se observa en otras variedades del español y en otras lenguas, entre otros hombre en español y home en catalán en España (Cuenca y Torres, 2008), güey en México (Kleinknecht, 2013), mate en Australia (Rendle-Short, 2009) y dude en Estados Unidos (Kiesling, 2004).

\footnotetext{
${ }^{1}$ Adoptamos la ortografía etimológica plena del término "huevón", tal como aparece en diccionarios y trabajos lingüísticos. Sin embargo, se suele pronunciar parcial o altamente reducido, de acuerdo con los rasgos fonéticos del estilo coloquial chileno (Rojas, 2012: 161), por lo que se escribe generalmente como 'weón' y 'wn' en canales escritos informales.

${ }^{2}$ Foros informativos informales en internet destacan también el uso frecuente de huevón en Chile: tiene una propia página en Wikipedia; en Forum.Wordreference se comentan entre otros: "As a chilean, I can say that that word is pretty much used. Every time you hear a chilean person, you'll hear this at the end of every sentence. [...] "huevon" is so current in Chile that it means almost nothing". (consulta: 16/01/13)
} 
A pesar de estas particularidades, solo tres estudios se han dedicado a la caracterización del fenómeno chileno. Sáez-Godoy (1983) examina, con base en una encuesta a 22 informantes nativos de distintas partes de Chile pero residentes en Alemania tras el golpe de Estado de 1973, en qué medida los hablantes conocen y cómo interpretan las diferentes derivaciones y construcciones a partir de hueva. Portocarrero (1998) discute el origen histórico y etimológico de la familia léxica de hueva y sus usos variados, significados, contextos y valoraciones actuales. Este inventario se basa en 'la mera competencia lingüística del autor' y se escribe como un ensayo popularizado y paródico (Portocarrero, 1998: 12). Finalmente, Rojas (2012) explora el proceso de gramaticalización de huevón de sustantivo a MDD a partir de un corpus $(n=1056)$ de periódicos nacionales, obras literarias, entrevistas, páginas electrónicas y foros de Internet recogido entre 2004 y 2009. Las contribuciones de Sáez-Godoy y Portocarrero son de carácter lexicográfico y se basan en un método intuitivo y/o evaluativo, i.e. sin el respaldo de usos concretos. Rojas parte de un análisis de usos concretos y describe los rasgos semánticos, sintácticos y fonéticos de huevón. Sin embargo, no considera factores pragmáticointeraccionales, sociolingüísticos y situacionales.

El presente trabajo propone atender estas deficiencias analizando instancias de huevón en conversaciones espontáneas cotidianas informales clasificadas según 22 parámetros lingüísticos y extralingüísticos. Como objetivo principal se propone la valoración semántica y pragmática de la FdT. ¿Qué significados tiene huevón? ¿Su uso implica alguna valoración negativa conservada de su significado original de insulto? Segundo, ¿en qué medida su uso es común o aceptable en conversaciones informales cotidianas teniendo en cuenta factores extralingüísticos como la edad, el sexo, la relación y jerarquía de los interlocutores, el marco interaccional y la meta comunicativa? Verificamos asimismo el lazo entre el uso de huevón y la (des)cortesía interaccional.

El análisis se desarrolla en tres fases: se estudia la etimología y los valores semánticos de huevón (Sección 2), se describe la naturaleza de los datos y parámetros considerados (Sección 3) y se discuten los rasgos más destacados del análisis a partir de resultados cuantitativos y cualitativos (Sección 4). 


\section{Estado de la cuestión}

El término huevón se forma mediante la raíz sustantiva "huev-" (del latín sg. ovum>huevo, pl. ova>hueva/huevos) y el sufijo aumentativo “-ón”. "Huevo", cuyo significado básico es "célula precedente de la unión del gameto masculino con el femenino en la reproducción sexual de animales y plantas', también puede referir a 'testículo' (Clave, 1996: 962). La derivación adjetival "huevón” literalmente significa ‘que tiene testículos de gran tamaño' (Rojas, 2012: 153). Sin embargo, este significado etimológico de huevón se perdió por completo, como ya atestigua Sáez-Godoy (1983), para ir proliferando en usos y expresiones figurados a partir de sucesivos desplazamientos semánticos (Rojas, 2012: 153).

La bibliografía atribuye tres significados básicos a la FdT huevón. El primer significado corresponde a 'de escasa capacidad intelectual' o 'tonto'. Con este significado, funciona de insulto (1):

(1) Hacia compañero: Esa huevá ${ }^{3}$ no se graba ahí, huevón. Esa huevá no es sonido, huevón. (Gen2.M $)^{4}$

El segundo significado es más general y simplemente refiere a 'persona, individuo (masculino)'. Puede funcionar como referencia a una persona (2) o como FdT (3):

(2) Hacia amigo: Yo soy un huevón- soy un huevón honrado, huevón. (Gen4.M)

(3) Hacia amigo: Ya huevón, amárratelo acá. (Gen4.M)

En la función de vocativo como en (3), huevón puede intercambiarse con otros títulos amistosos o neutros como amigo, compadre o tú (Sáez-Godoy, 1984: 143; Portocarrero, 1998: 22).

El tercer significado básico se describe como "una marca de fin de frase" (Sáez-Godoy, 1983: 143), "un elemento fático" (Portocarrero, 1998: 22) o un "marcador de la intención de mantener la atención del interlocutor" (Rojas, 2012: 162). Funciona como MDD y constituye según Rojas (2012: 162) el último paso en el proceso de gramaticalización de la palabra. Este uso pertenece a la clase de los enfocadores de la alteridad, como propone la clasificación de los MDD de Martín Zorraquino y Portolés (1999), tal como por ejemplo hombre, bueno, vamos, mira y oye (Rojas,

\footnotetext{
${ }^{3}$ Huevá(da): f. vulgar. 'Cosa o asunto' (Academia Chilena de la Lengua, 2010)

${ }^{4}$ Para la explicación de los códigos de los fragmentos, véase la metodología (infra §3.1).
} 
2012: 156). Su significado semántico se sitúa en el nivel discursivo y metalingüístico y ejerce dos funciones principales:

a) Mediante su función fática garantiza y refuerza las relaciones sociales entre los interlocutores y llama la atención del oyente sobre el mensaje. Sintácticamente, afecta al enunciado entero (4).

b) A veces, mediante una función procedimental adicional, da instrucciones semánticas sobre el procesamiento de los miembros del discurso (Martín Zorraquino y Portolés, 1999: 4072). Sintácticamente, es delimitador entre enunciados (Rojas, 2012: 160) (5).

(4) Hacia amigas: Después la sacaban a bailar. Huevón, y así un rostro, así de un metro y después se quería ir, porque le dolía la guata, no sé. (Gen2.F)

(5) Hacia hermano: Miranda tiene unas canciones bacanes ${ }^{5}$ po huevón. Y son pegajosas po huevón, no sé qué tiene ese grupo huevón. (Gen3.M)

Pese a que en el ejemplo (4) huevón se encuentra en la posición por excelencia de un vocativo (i.e. en yuxtaposición a la izquierda), se excluye la interpretación 'persona', pues la frase se dirige a varias personas y además son mujeres. Tanto en (4) como en (5) se procura llamar la atención del oyente sobre el mensaje. En (5), además, los tres huevón señalan el límite entre los tres enunciados. Entre los enfocadores de la alteridad, huevón se asemeja mucho a hombre, que también resulta de un proceso de gramaticalización a partir de un sustantivo empleado como vocativo (Martín Zorraquino y Portolés, 1999: 4172). Se le asignan al MDD hombre las funciones pragmáticas siguientes (ibíd.: 4173-4174):

a) Refuerza las relaciones sociales entre los interlocutores, imprimiendo un tono amistoso y de complicidad a la conversación

b) Atenúa la expresión de la disconformidad con lo dicho por el oyente e incluso introduce efectos paliativos para calmar su posible enfado

c) Atempera un enunciado directivo para evitar que su actitud exhortativa o imperativa molesten al oyente

d) Provoca un cierto tono chistoso, alegre, etc., para señalar la sorpresa que algo provoca

Verificaremos en nuestro análisis en qué medida huevón manifiesta estas mismas funciones. El tercer significado, que llamamos 'atención', incorpora este conjunto de funciones fáticas y

\footnotetext{
${ }^{5}$ Bacán: adj. 'Muy bueno o muy bien' (Academia Chilena de la Lengua, 2010)
} 
procedimentales. Recalcamos que el término no sólo alude al significado 'llamar la atención', sino que lo proponemos como término más bien abstracto que engloba todas las posibles funciones de huevón como MDD.

Además de los tres significados básicos, Rojas (2012: 155) menciona que existe una fase semántica intermedia entre 'tonto' y 'persona'. Así, huevón sigue siendo un insulto hacia el oyente, pero no respecto a su falta de inteligencia sino a una actitud o un comportamiento más general desaprobado por el hablante. Este uso de vocativo se denominará 'comportamiento desaprobado’ ((6) y (7)):

(6) Compañero 1: Tú no mataste Freezer ey.

Compañero 2: Sí, lo maté.

Compañero 1: No, porque Goku se puso a pelear con Freezer y después Goku se se esquivó un platillo que le disparó Freezer y después el platillo se le devolvió a Freezer y le cortó y lo cortó por la mitad.

Compañero 2: Sí poh, murió.

Compañero 1: Ya poh, pero tú no lo mataste poh.

Compañero 2: Yo lo maté.

Compañero 1: Tú no le disparaste ese platillo.

Compañero 2: Ah, huevón, yo lo maté. Yo estuve en esa pelea, yo lo peleé. (Gen1.M, en un furgón escolar)

(la encargada de la grabadora se dirige a un compañero de curso, quien juega con la grabadora haciéndose de periodista, preguntando ahora a otro compañero sobre su supuesto trabajo en la prostitución y distintos tipos de sexo)

(7) Hacia compañero: No hagái eso, huevón, qué vergüenza. (Gen2.F a Gen2.M)

Al distinguir el significado original 'tonto' de otros usos ofensivos semánticamente más generales se revela el primer paso de la gramaticalización de huevón.

Finalmente, consideramos un quinto significado como 'contexto negativo'. Cuando huevón funciona de MDD, se rodea a menudo de un aspecto despectivo, o bien en el contenido del enunciado/intervención o bien en la entonación del hablante ((8), (9):

(8) Amiga 1: (sobre Google Earth) Salen unas personas saliendo de un motel. ¿Cachái? O sea, cero privacidad. Amiga 2: Sí, huevón, cero respeto, huevón. (Gen2.F)

(9) Hacia novia: (sobre cómo ella va vestido) Tápate esas cosas, parecíh tonta, huevón. (Gen3.M a Gen3.F)

Se podría interpretar este significado como un MDD, pero con un grado menor de abstracción semántica que ‘atención’ dado que mantiene una connotación negativa. 
Resultará útil tratar por separado los significados con valor negativo de los con valor neutro o positivo por un lado, y los tratamientos al oyente directos (como vocativo) de los indirectos (como MDD) (directo con 'tonto', 'comportamiento desaprobado' y 'persona') por otro lado, por su implicación en la cortesía y el riesgo de amenazar o no la imagen social del hablante/oyente. Sin embargo, admitimos que los cinco significados no siempre son fáciles de separar: al igual que entre otros hombre y home en español y catalán, "su significado se organice como una cadena de significados o categoría radial [...] como sucede en general con las palabras polisémicas" (Cuenca y Torres, 2008: 251). Aclaremos nuestra clasificación, que se basa en dos criterios formales. El primer criterio es la +/-concordancia en género y número de huevón (masc. sg.) con su referente. Así, calificamos imposible el uso de huevón como vocativo cuando no hay concordancia, como en los fragmentos (4) (fem. pl.) y (8) y (9) (fem. sg.). Según el segundo criterio se verifica si huevón se deja reemplazar por otro término. Si la frase se hace ilógica semánticamente o se intuye como extraña o poco económica estilísticamente, es improbable el significado relacionado a este término insertado. He aquí tres pruebas en las que huevón se puede reemplazar, respectivamente:

a. Por tonto: indica el significado 'tonto'. P.ej. en uno de los dos usos de $(1)^{6}$.

b. Por una calificación negativa determinada que no infiera 'poco inteligente': indica el significado ‘comportamiento desaprobado'. P.ej. 'pesado’ o ‘reparón’ en (6), 'desubicado’ o 'indecente' en (7).

c. Por un nombre propio: indica un uso como vocativo. P.ej.: en uno de los dos usos de (1), (3), (6), (7). Sirve sobre todo para distinguir el valor 'persona' de los usos como MDD.

\section{Metodología}

\subsection{Los datos}

Los datos provienen de audio-grabaciones efectuadas por la autora en Iquique, una ciudad chilena norteña, entre julio y octubre del 2012 y se recogen en el Corpus de Conversaciones Espontáneas del Español Chileno, Iquique (CCEEC-I) (Helincks, en preparación). En general, se trata de

\footnotetext{
${ }^{6}$ Es dudoso que funcione de 'tonto' en ambos usos del fragmento, dado que la repetición del vocativo tan seguido dentro de la intervención es poco económica y redundante.
} 
conversaciones surgidas naturalmente en seis ámbitos: dos ámbitos privados 'familia' y 'amigos', y cuatro ámbitos públicos 'reunión social/religiosa', 'colectivo'7, 'furgón escolar' y 'peluquería'. Las situaciones grabadas son variadas ya que son de diferentes furgones escolares, reuniones sociales, peluqueras, familias y grupos de amigos. La lingüista-grabadora estaba presente como participante u observadora en una de las situaciones 'familia' y en todos los ámbitos públicos salvo las reuniones sociales. Respecto de la relación entre la investigadora y los participantes estudiados, en la familia la investigadora participa como la novia de un miembro de la familia, en el colectivo es un participante-pasajero, en el furgón escolar y la reunión religiosa participa como una invitada tras haber sido introducida como investigadora y en la peluquería observa desde un sillón mientras unas peluqueras tienen puesta una grabadora. La mayoría de los participantes se informaron de ser grabados. Solo en los ámbitos 'colectivo' y 'furgón escolar', la grabación es semi-secreta: el colectivero y el/la conductor(a) de furgón están al tanto de la grabación, mientras que los clientes y niños de furgón no. En las reuniones sociales y los ámbitos privados, la grabación es efectuada por amigos y conocidos de la investigadora. Estas personas casi siempre informaron a los demás participantes de la presencia de la grabadora ${ }^{8}$.

El corpus tiene un total de 33h35min de grabación o 322.872 palabras. Participan 223 hablantes de los que, según la cantidad de palabras, un 8\% son niños (0-14 años), un 23\% son jóvenes (1525 años), un $38 \%$ son adultos (26-55 años) y un $29 \%$ son mayores ( $\geq 56$ años). Los sexos están bien equilibrados con un $54 \%$ de hombres y un $46 \%$ de mujeres. En cuanto a la proporción de los ámbitos un $29 \%$ se expresa en 'reunión', un $27 \%$ en 'familia', un $21 \%$ en 'amigos', un $9 \%$ en 'peluquería', un $8 \%$ en 'furgón escolar' y un 6\% en 'colectivo'. Los códigos añadidos a los fragmentos informan sobre la generación y el sexo de los interlocutores, es decir Gen1=niño, Gen2=joven, Gen3=adulto, Gen4=mayor, $\mathrm{M}=$ masculino y $\mathrm{F}=$ femenino. Si solo se añade un código (y no p.ej. 'Gen2.M a Gen2.F'), los mismos rasgos valen para el hablante y el oyente.

\footnotetext{
${ }^{7}$ El colectivo es un sistema de transporte público común de Chile. Es similar al taxi pero puede estar varios clientes a la vez que se desconocen.

${ }^{8}$ Varios indicios a lo largo de las grabaciones indican que la presencia de la grabadora influye poco en la naturalidad del habla de los participantes: p.ej. las actividades y temáticas cotidianas de cada ámbito no se interrumpen y a veces una exclamación y/o comentario de sorpresa al final de una grabación indica de haber olvidado de apagar la grabadora. Una excepción es una conversación entre compañeros de curso. Aquí, la presencia de la grabadora incita a un participante a imitar una entrevista formal usando la grabadora como un micrófono (supra el fragmento (7)).
} 
El presente estudio enfoca solo el uso del sustantivo huevón usado como FdT (de vocativo y MDD). No discute los demás usos derivados, ni el uso de huevón como referencia a una persona. Tampoco se discute el equivalente femenino huevona que también puede funcionar como FdT $^{9}$. En su totalidad el corpus contiene 715 instancias de huevón como FdT.

\subsection{Los parámetros de análisis}

El cuadro 1 resume los 22 parámetros y sus correspondientes valores examinados.

Cuadro 1: Los parámetros del análisis

\begin{tabular}{|c|c|c|c|}
\hline \multicolumn{2}{|c|}{ Tipo de parámetro } & Parámetro & Valores \\
\hline \multirow{4}{*}{\multicolumn{2}{|c|}{ Social }} & generación de hablante & niño, joven, adulto, mayor \\
\hline & & sexo de hablante & masculino, femenino \\
\hline & & $\begin{array}{l}\text { generación de oyente(s) (para con } \\
\text { hablante) }\end{array}$ & $\begin{array}{l}\text { misma, }>1 \text { gen., }>2 \text { gen., }<1 \text { gen., }<2 \text { gen., } \\
\text { varias }\end{array}$ \\
\hline & & sexo de oyente(s) (para con hablante) & mismo, opuesto, ambos \\
\hline \multirow{3}{*}{\multicolumn{2}{|c|}{ Relacional }} & relación existencial de proximidad & íntimo, conocido, desconocido \\
\hline & & relación jerárquica & igualitaria, superior, inferior \\
\hline & & rol funcional & (29 roles específicos, p.ej. padre, cliente) \\
\hline \multirow{2}{*}{\multicolumn{2}{|c|}{ Situacional macro }} & marco interaccional & cotidiano, no cotidiano \\
\hline & & tono discursivo & formal, informal \\
\hline \multirow{4}{*}{\multicolumn{2}{|c|}{ Situacional micro }} & finalidad comunicativa predominante & transaccional, interpersonal \\
\hline & & grado de problematicidad temática & bajo, medio, alto \\
\hline & & aceptación lingüística/social & meta compartida, meta no compartida \\
\hline & & humor & sí, no \\
\hline \multirow[t]{9}{*}{ Ling. } & Fonético & realización fonética & plena, débil, muy débil \\
\hline & Sintáctico & posición sintáctica & inicial, media, final \\
\hline & \multirow[t]{2}{*}{ Léxico } & colocación con FdT pronominal & usted, tú, vos, n.a. \\
\hline & & colocación con $2^{\mathrm{a}}$ p.sg. del verbo & ustedeo, tuteo, voseo, n.a. \\
\hline & Semántico & significado semántico-pragmático & $\begin{array}{l}\text { tonto, comportamiento desaprobado, persona, } \\
\text { atención, contexto negativo }\end{array}$ \\
\hline & \multirow[t]{4}{*}{ Pragmático } & modalidad discursiva & tratamiento directo, cita reproducida \\
\hline & & acto de habla & $\begin{array}{l}\text { asertivo, compromisorio, declarativo, } \\
\text { directivo, expresivo }\end{array}$ \\
\hline & & actividad de imagen & mantener, mitigar, valorar, amenazar \\
\hline & & reacción de oyente & sentido, ofender, defender, seguir, afirmativo \\
\hline
\end{tabular}

${ }^{9}$ La forma huevona tiene características semántico-pragmáticas, sociolingüísticas y situacionales considerablemente diferentes de las de huevón como para merecer una investigación comparativa detallada. Sin embargo, por falta de espacio, no podemos incluir esta comparación aquí. 
Aclaremos algunos parámetros y valores. Primero, respecto de la generación y el sexo de oyente(s), los valores 'varias' y 'ambos' refieren a situaciones de dos o más oyentes de generaciones y/o sexos diferentes. Segundo, adoptamos los parámetros problematicidad temática y aceptación lingüística/social de Briz (2004), quien los define como "filtros evaluadores" de la cortesía en un discurso. Problematicidad temática refiere al grado de controversia potencial de ciertas temáticas: "temas polémicos o delicados en los que se produce un choque o conflicto individual y social, entre lo que es y lo que debería ser: el racismo, la homosexualidad", o temas personales en relación con las emociones o el derecho del yo a la intimidad o a decidir (Briz 2005: 72) (vea una problematicidad temática 'alta' p.ej. en (7), (9) y (15)). El segundo parámetro de Briz identifica el (des)acuerdo entre los interlocutores, es decir si la meta comunicativa del hablante coincide con o se enfrenta a las metas del oyente (2004: 84) (vea una 'meta no compartida' p.ej. en (1), (6), (13) y (15)). Ambos parámetros pueden revelar si enunciados implican tensión entre los interlocutores, por lo que contribuyen a determinar el valor semánticopragmático y una (des)cortesía potencial en el uso de huevón.

Tercero, para la realización fonética adoptamos la distinción tripartita de Rojas (2012: 161) entre una pronunciación 'plena' [gweón], 'débil' [won] o [wən] y 'muy débil' [on] o [ən]. Cuarto, el parámetro modalidad discursiva señala si la FdT se dirige a los participantes involucrados en la grabación o si expresa más bien una FdT reproducida en forma de cita (p.ej. "Y yo les dije <Ya poh huevón>, pero yo igual estaba asustado"). Finalmente, introducimos el parámetro reacción de oyente como una manera de medir objetivamente cómo el oyente evalúa lo dicho por el hablante. Distinguimos cinco valores de reacciones del oyente a lo que el hablante acaba de decir. Los primeros (a) 'sentido', (b) 'defender' y (c) 'ofender' implican reacciones negativas: (a) un marcado silencio que sugiere sensibilidad, (b) una respuesta defensiva y (c) una respuesta ofensiva. La reacción (d) 'seguir' representa neutralidad. Finalmente, la reacción (e) 'afirmativo' refiere a una respuesta positiva del oyente, por ejemplo mediante una afirmación, risa o elaboración de la idea del otro.

El método del análisis es sintético: en vez de enumerar los resultados de los 22 parámetros por separado, incorporamos los resultados más notables bajo cuatro hipótesis fundamentales en cuatro subapartados. Cada hipótesis se argumenta mediante resultados cuantitativos y cualitativos de diferentes parámetros. 


\section{Rasgos sociopragmáticos de huevón en Chile}

\subsection{La importancia de confianza, informalidad y rol funcional}

El grado de confianza de los interlocutores y la informalidad de la situación suelen influir considerablemente en la variación estilística del lenguaje. También en el caso de huevón estos dos factores se confirman. En el corpus, su influencia se evidencia en primer lugar en la distribución global de huevón sobre los seis ámbitos, como el gráfico 1 (uso por 100.000 palabras) muestra:

Gráfico 1: La influencia de la intimidad relacional e informalidad situacional sobre el uso de huevón

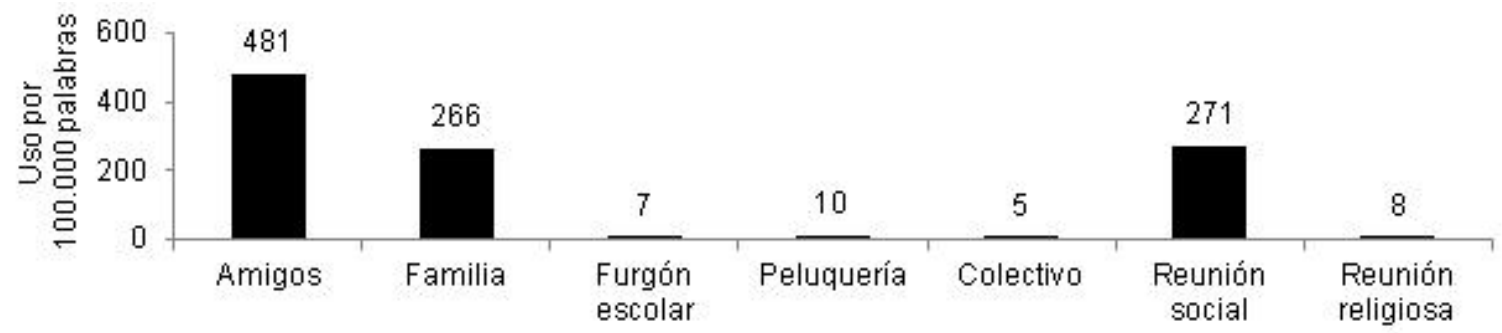

Cabe notar que desdoblamos el ámbito 'reunión' en uno 'social' y otro 'religioso' por la relación de proximidad diferente de los participantes en ellos (i.e. resp. 'íntimos' y ‘conocidos'). El uso de huevón claramente predomina en los ámbitos 'amigos', 'familia' y 'reunión social', es decir los de mayor confianza e intimidad, y apenas aparece en los demás ámbitos.

El uso de huevón también se relaciona con la informalidad. Pese a que todos los ámbitos sean informales y cotidianos, está claro que 'amigos' y 'familia' caracterizan el grado más alto. Los resultados de los parámetros colocación con $2^{a}$ p.sg. del verbo y colocación con FdTs pronominales contribuyen a esta tesis. Estos parámetros señalan si la persona que dirige huevón a alguien, le dirige también una forma de ustedeo, tuteo o voseo en el co-texto de huevón (i.e. de treinta segundos previos a diez segundos posteriores). Cabe comentar que el español chileno incluye una forma de tratamiento híbrida, el 'voseo mixto verbal': combina el pronombre sujeto tú con formas verbales voseantes, p.ej. tú hablái, tú comí(s) y tú decí(s) (Torrejón, 1986). De las 220 colocaciones verbales observadas, el $83 \%$ es voseante. De las 65 colocaciones pronominales, el $80 \%$ constituye la forma tú. El porcentaje restante de ambos parámetros se divide igualmente 
sobre las otras dos formas, es decir ustedeo/tuteo y usted/vos. Efectivamente, el voseo verbal y mixto chilenos suelen prevalecer en el habla coloquial y relaciones solidarias y de confianza (Helincks, 2012). Esta misma condición de un alto grado de proximidad e informalidad caracteriza los MDD hombre/home (Cuenca y Torres, 2008: 238), güey (Kleinknecht, 2013: 151) y dude (Kiesling (2004: 292, 298), mientras que mate (ya) se extiende a ámbitos más formales (el trabajo, instituciones como la radio y el parlamento) e interacciones entre desconocidos (RendleShort, 2009: 266).

Aun así, la intimidad e informalidad no logran explicar todos los casos de variación situacional de huevón. Así, su frecuencia en 'amigos' supera notablemente las de 'familia' y 'reunión'. Esta diferencia se reafirma en dos aspectos situacionales más. En primer lugar, se nota una diferencia en el comportamiento de un mismo hablante en ámbitos diferentes, en concreto entre 'amigos' y 'familia'. Tres personas del corpus aparecen en ambos ámbitos y hablan suficientemente -ofrecen al menos 2000 palabras-en ambos y utilizan huevón al menos en uno de ellos: una mujer de 26 años y dos hombres de 31 años y 74 años. Cotejamos su uso de huevón en el gráfico 2 (uso por 100.000 palabras).

Gráfico 2: La influencia del rol interaccional en el uso de huevón

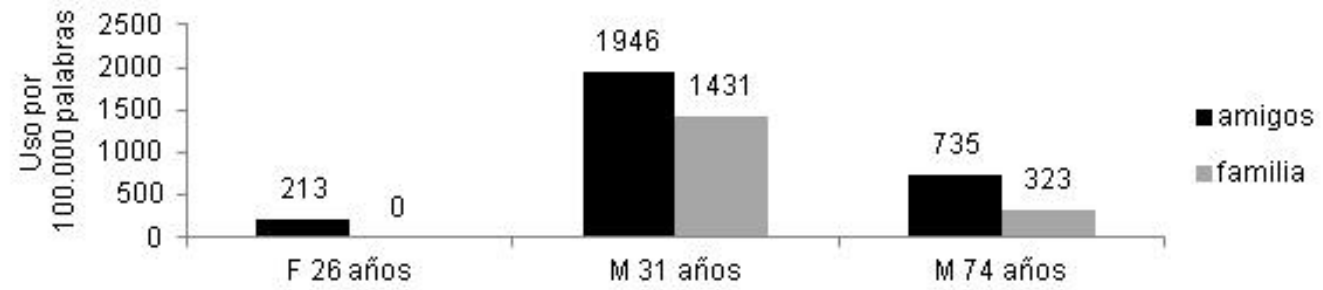

Los tres hablantes concuerdan en un uso reducido de huevón entre familiares para con su uso entre amigos. Esta observación sugiere que la jerarquía o el rol funcional/interaccional desempeña un papel determinante en la selección de huevón. En la familia existe una jerarquía ya que padres, tíos y abuelos son (mucho más) mayores que hijos, sobrinos y nietos. Además, aquellos ejercen un rol de responsables y educadores mientras que de estos se supone un comportamiento de buena educación. Aparentemente, estos roles permiten menos expresiones de huevón que la relación completamente igualitaria de amigos.

El segundo aspecto que reafirma una variación situacional se muestra en el uso de huevón desequilibrado a lo largo de una de las reuniones sociales. Se trata de un encuentro entre seis 
hombres y dos mujeres mayores de 70 años de la junta directiva de una organización de adultos mayores. Se reúnen mensualmente para discutir asuntos pendientes y después suelen tomar té juntos charlando de temas cotidianos. El discurso de la parte 'oficial' de 1h30min forma parte del ámbito 'reunión' y la charla recreativa de oh37min se incorpora en el ámbito ‘amigos'. El gráfico 3 visualiza la frecuencia de uso de huevón sobre el encuentro por cada 15 minutos.

Gráfico 3: El uso de huevón a lo largo de la primera reunión social con un parte 'oficial' y una parte recreativa

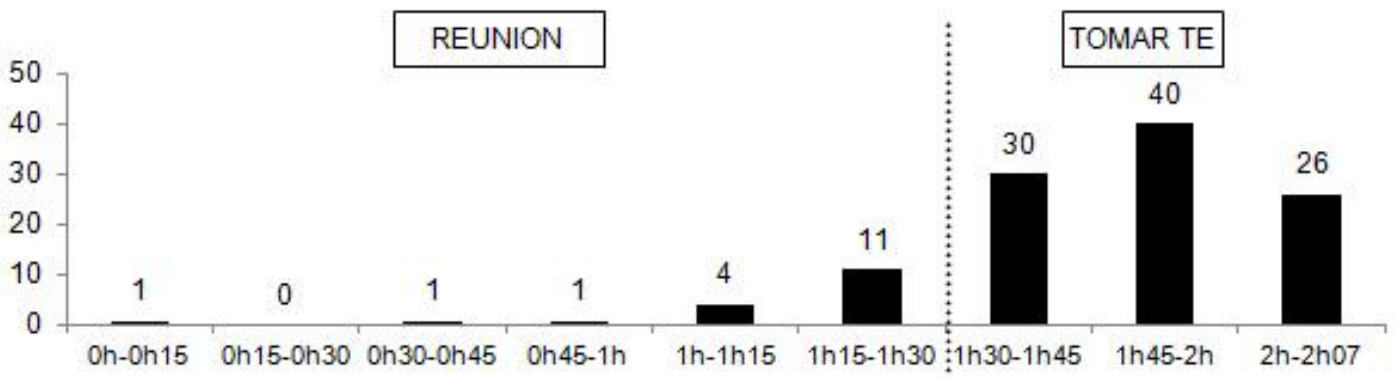

El gráfico indica claramente que apenas se expresa la FdT durante la propia reunión, mientras que sí surge frecuentemente cuando están tomando té. Por consiguiente, el contenido de la conversación, o más bien la finalidad comunicativa, influye claramente en la selección de la forma por los hablantes. En la primera parte, los temas son más serios o transaccionales y los participantes representan un rol institucional de miembro ejecutivo que se prioriza a su rol de amigo. La mayor responsabilidad y formalidad que requiere el primer rol excluye un uso abundante de huevón.

Es interesante comparar estos datos con los de la segunda reunión social del corpus que representa una junta directiva similar con rasgos sociales y relacionales casi idénticos, salvo que no asisten mujeres en ella. Aun así, huevón aparece mucho más aquí, con 368 instancias frente a 92 en la reunión anterior.

Gráfico 4: El uso de huevón a lo largo de la segunda reunión social sin pausa

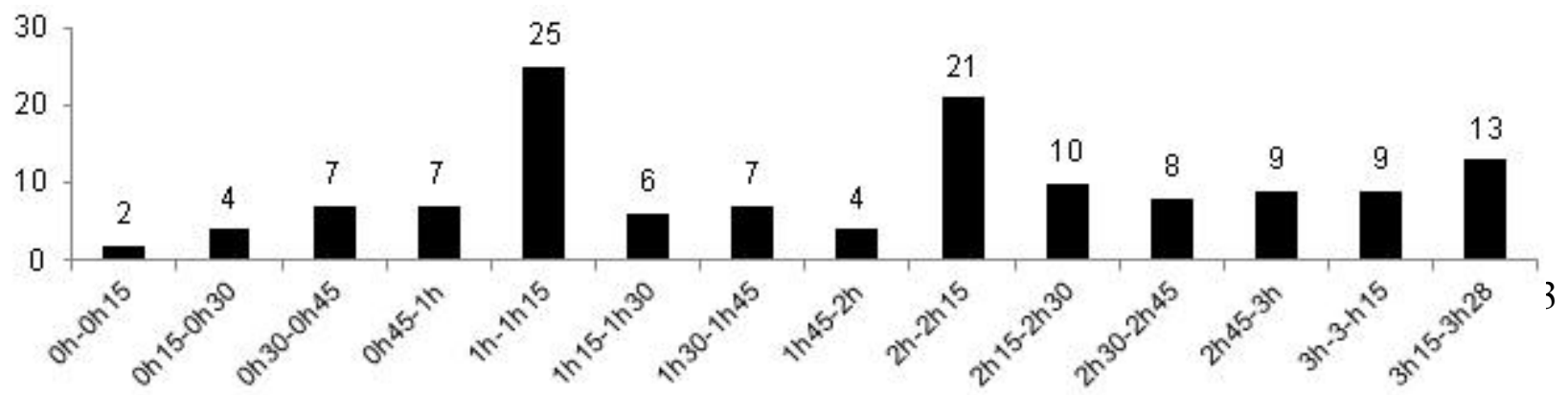


Esta reunión dura 3h28min sin pausa para tomar té. Como se ve en el gráfico 4 , los usos de huevón se reparten más proporcionadamente, con una concentración mayor en dos fragmentos. En el primero de estos fragmentos, los miembros se desvían de los asuntos organizativos y se extienden sobre un suceso personal. En cuanto a los demás usos, huevón funciona sobre todo como MDD que atenúa expresiones de disconformidad (infra: §4.4) y como MDD solidario y de complicidad en breves comentarios secundarios a un asunto. No obstante, estas dos funciones de huevón también predominan en la primera junta y ambas juntas presentan bastantes discusiones tensas o polémicas para que estimularan una frecuencia similar del huevón atenuador. Por lo tanto, ¿cómo se explica su mayor frecuencia en la segunda junta? En general, esta junta parece irradiar una atmósfera más relajada. El parámetro colocación con $2^{a}$ p.sg. del verbo ya lo sugiere: de los tres contextos 'primera junta, parte oficial', 'primera junta, parte recreativa' y 'segunda junta', este último tiene el porcentaje más bajo de ustedeo y más alto de voseo ${ }^{10}$. Una posible explicación para esta atmósfera diferente es la participación de mujeres -solo asisten mujeres a la primera junta-, dado que esto influye en el uso de huevón (infra §4.3). En todo caso, entre hombre-mujer los hablantes se tratan casi consecuentemente de usted, mientras que entre hombre-hombre y mujer-mujer prevalecen el tuteo y voseo.

\subsection{Huevón no es solo una expresión de jóvenes}

Es muy general la presuposición de que huevón sea un término típico del lenguaje juvenil. Esto se evidencia tanto en comentarios de hablantes nativos legos ${ }^{11}$ como en trabajos lingüísticos (Albelda, 2008: 110; Jørgensen, 2011: 135). Similarmente, Kiesling (2004: 299) argumenta respecto de la FdT dude que su carácter nonconformista y de "cool solidarity" atrae a jóvenes y que esta atracción "flattens out as teenagers become older". Sin embargo, su estudio solo se basa en observaciones y encuestas realizadas en el $91 \%$ por y con personas menores a los 30 años (ibid.: 302). Además, cabe señalar que investigadores se encuentran en general en una posición

\footnotetext{
${ }^{10}$ E primer contexto muestra un $34,5 \%$ de ustedeo vs. un $38,9 \%$ de tuteo vs. un $26,6 \%$ de voseo, el segundo un $14,7 \%$ vs. un $62,3 \%$ vs. un $23 \%$ y el tercero un $7,8 \%$ vs. un $48,1 \%$ vs. un $44,1 \%$.

${ }^{11}$ Nos basamos en las respuestas de contactos universitarios chilenos y nativos en el foro electrónico Forum. WordReference a nuestra pregunta de interpretar el uso de huevón en Chile.
} 
social inferior, es decir de (mucho) menor edad, cuando observan/entrevistan a personas realmente mayores.

El presente análisis rebate esta suposición, sugiriendo prudencia ante la demasiada obviedad de ella. El análisis demuestra claramente que todas las generaciones recurren al uso de huevón. Aún más, en cifras absolutas, los adultos y mayores presentan el uso más frecuente, con el $40 \%$ $(n=286)$ y el 39\% ( $n=277)$ resp., mientras que los jóvenes y niños lo usan en un 20\% (n=145) y un $0,6 \%(n=4)$ resp. de los casos analizados ${ }^{12}$. Los resultados destacan aún más cuando se estiman según el número de palabras emitidas. Los adultos producen el 38\% del total de palabras del corpus, los mayores el 29\%, los jóvenes el 23\% y los niños el 9\%. Ahora, al calcular el uso de huevón por cada 100.000 palabras, los mayores presentan la densidad de uso más alta, un 40\%, seguidos de los adultos (32\%), jóvenes (26\%) y niños (2\%).

Cabe recalcar que con esto no sugerimos que los mayores sean los usuarios principales del término huevón. La composición del corpus así como factores contextuales adicionales explican la diferencia de frecuencia entre los grupos generacionales. Por ejemplo, los jóvenes y adultos se presentan en ámbitos más variados que los mayores, mientras que una parte considerable del habla de estos últimos ocurre en el ámbito 'reunión social'. No obstante, las cifras absolutas y la densidad de uso demuestran que en determinadas circunstancias los mayores son igualmente capaces de producir una alta frecuencia de huevón.

\subsection{Utilizar huevón es un asunto de hombres}

Rojas (2012: 157) señala que "al menos algunos hablantes perciben que el uso de huevón como enfocador de la alteridad es más propio de hombres que de mujeres”. El lingüista vincula esta diferencia a la mayor inclinación de hombres a usar palabras groseras y lenguaje no estándar, es decir formas de prestigio encubierto (ibid.: 157-158).

Los datos comprueban esta hipótesis en cuatro aspectos. Primero, la frecuencia general revela que los hombres expresan 615 de los 715 casos de huevón, lo cual corresponde a una proporción de uso (por 100.000 palabras) del 86\%. Segundo, hombres expresan huevón en una mayor diversidad de roles funcionales. El corpus distingue 29 roles sobre todos los ámbitos. Hombres

\footnotetext{
${ }^{12}$ Tres instancias de huevón son producidas por hablantes de edad desconocida.
} 
usan huevón en todos los roles, mientras que mujeres lo usan solo en cinco: amiga, compañera, hermana, novia y empleada (peluquera en este caso). En cambio, no lo usan en por ejemplo su rol de madre, esposa, hija, tía, prima o cliente (vea también supra gráfico 2). Aunque ambos hombres del gráfico 2 también reducen su uso de huevón en el ámbito 'familia' para con 'amigos', no lo suprimen por completo.

Tercero, relacionado al parámetro de roles, el grado de proximidad relacional también señala una actitud diferente entre los sexos. Mientras que las mujeres usan huevón (casi) exclusivamente en roles íntimos, hombres lo dirigen en varios casos $(\mathrm{n}=10)$ a un mero 'conocido' o aún 'desconocido' (de la misma generación o una generación menor) en la calle, una tienda o un bar. La FdT mate (Rendle-Short, 2009: 253) revela un uso/valoración similar: las mujeres encuestadas comentan que usan mate sobre todo "as a friendly term or as a term of endearment, used within a relaxed, informal or casual context" (contestado en un 45,8\%), mientras que para hombres esta es solo la segunda razón (un 28,4\%) después de "out of habit" (un 34\%) y junto a otras razones como "in place of a name" (un 18,2\%) y "Australian term" (un 16,4\%).

Cuarto, hombres y mujeres difieren en el uso semántico-pragmático de huevón. La diferencia se halla sobre todo en el uso de los tipos de MDD 'contexto negativo' y 'atención'. Cuando mujeres utilizan huevón, este se rodea mucho más de un contexto negativo: con mujeres, un 55\% ( $\mathrm{n}=55$ ) de casos expresa 'atención' y un 36\% ( $\mathrm{n}=36)$ 'contexto negativo', frente a un 76\% ( $\mathrm{n}=466)$ y un $15 \%$ ( $\mathrm{n}=93$ ) resp. de los usos de huevón emitidos por hombres (vea resultados similares para dude, Kiesling, 2004: 285). En otras palabras, mujeres parecen considerar huevón todavía más como una expresión con connotación negativa.

Por otro lado, el análisis señala que hombres no solamente utilizan huevón más frecuentemente, sino que también lo dirigen principalmente a hombres, mientras que mujeres lo dirigen a ambos sexos de medida similar. Sólo el 12\% ( $\mathrm{n}=73)$ de los usos por hombres es dirigido a una mujer o a ambos sexos en caso de varios oyentes ${ }^{13}$. La misma tendencia se señala para dude (Kiesling, 2004: 285, 287) y mate (Rendle-Short, 2009: 253), mientras que home en catalán parece darse de igual modo entre y a ambos sexos (Cuenca y Torres, 2008: 248, nota 8).

\footnotetext{
${ }^{13}$ Somos conscientes de que estos datos cuantitativos no son completamente fiables por la composición del corpus, pues muchas interacciones tienen lugar entre $\mathrm{u}$ hombres-hombres o mujeres-mujeres.
} 
El fragmento (10) corrobora esta actitud de hombres hacia huevón. El joven del fragmento presenta la proporción de uso de huevón más alta del corpus. Tiene 20 años y aparece en el ámbito 'familia' conversando principalmente con sus primos. Sin embargo, en una conversación de 18 minutos, charla con su primo y la novia de este primo, ambos de edad similar a él. A lo largo de los 18 minutos, el joven produce 46 instancias de huevón. Cuando el primo sale durante cinco minutos para llamar por teléfono y el joven solo charla con la novia de aquel, no expresa ningún huevón. Veamos una parte de la interacción en (10) (al inicio no está el primo).

(10)

\begin{tabular}{|c|c|}
\hline $\begin{array}{l}\text { Novia de primo: } \\
\text { Joven: }\end{array}$ & ¿Y qué pasó con tu celular así? \\
\hline Joven: & Ya poh, yo tenía uno y el Juanito cuando-, hace tiempo, \\
\hline $\begin{array}{l}\text { Joven: } \\
\text { Novia de primo: }\end{array}$ & $\begin{array}{l}\text { uno, la Javiera-, porque son dos poh. } \\
\text { Sí poh. }\end{array}$ \\
\hline $\begin{array}{l}\text { Joven: } \\
\text { Novia de primo: }\end{array}$ & prueba de conducir, fue a sacar la licencia, se le quedó allá. \\
\hline $\begin{array}{l}\text { Joven: } \\
\text { Primo: }\end{array}$ & Porque yo lo había dejado así \\
\hline oven: & libre porque- no ése es el celular que yo tenía. Y ya me he comprado este. \\
\hline oven: & a mí, por ejemplo, éste no me gusta en el sentido porque, escribo muchos \\
\hline $\begin{array}{l}\text { Joven: } \\
\text { Novia de pr } \\
\text { Primo: }\end{array}$ & Pero no me gusta esta cuestión. \\
\hline
\end{tabular}

Muchos de los usos de huevón del joven funcionan de MDD fáticos. Por lo tanto, en teoría se pueden dirigir tanto a un hombre como a una mujer. Es curioso que aunque sí puedan participar mujeres en la conversación, al usar huevón los hombres las parecen excluir de alguna manera. Con ello se confirma la aserción de Rojas (2012: 158): que huevón ha llegado a funcionar como un símbolo de identidad grupal interna de hombres.

\subsection{Utilizar huevón no suele ser descortés}

En nuestro corpus huevón apenas mantiene usos de su función básica de insulto (vocativo). De los cinco significados semántico-pragmáticos destacados, 'tonto' y 'comportamiento desaprobado' representan una minoría, con apenas resp. un $1,4 \%(n=10)$ y un $0,8 \%(n=6)$ de los casos analizados. Los valores 'persona' y 'contexto negativo' aparecen más frecuentemente, abarcando resp. el $8 \%(n=57)$ y el 17,6\% $(n=126)$ de los usos, mientras que 'atención' claramente 
predomina, cubriendo el 73,4\% (n=525) de los casos analizados. La FdT mexicana güey, cuyo origen semántico también es despectivo (>buey, expresando 'estúpido, idiota'), igualmente ha perdido esta connotación negativa gradualmente y hoy en día es general su uso como MDD (Kleinknecht, 2013: 142).

Como ya indicamos (supra §2.2), pragmáticamente el valor 'atención' se asemeja al valor de hombre como MDD en el español peninsular. Sin embargo, ambos difieren en que este último deriva de un sustantivo semánticamente neutro mientras que huevón no. Por lo tanto, cabe indagar si persisten en el uso actual del MDD huevón valores distintivos que en el uso de hombre. Para ello, es útil verificar no solo su significado semántico-pragmático, sino también el fin del hablante (i.e. ¿qué meta comunicativa quiere lograr y cómo lo quiere lograr?) y la interpretación y evaluación del oyente. En otras palabras, ¿cuál es el efecto del uso de huevón en la interacción y para la relación de los interlocutores? Se analizan estos aspectos mediante los parámetros acto de habla, aceptación lingüística/social, actividad de imagen y reacción de oyente.

Huevón aparece en los cinco tipos de acto de habla con una proporción regular, es decir similar a su proporción en general en conversaciones informales cotidianas: predominan los actos asertivos (un 52,7\%, n=377), expresivos (un 34\%, $\mathrm{n}=244$ ) y directivos (un 11,6\%, $\mathrm{n}=83$ ). Respecto de la aceptación lingüística/social, huevón ocurre en enunciados de meta tanto compartida como no compartida con una proporción de 2:1. La distribución de las actividades de imagen que se combinan con huevón tampoco es llamativa: la estrategia de 'mantener' el equilibrio relacional aparece más (el 47,4\%, n=338), seguido de 'amenazar' (el 25,2\%, n=180), 'mitigar' (el 14,2\%, $\mathrm{n}=101$ ) y 'valorar' (el 12,6\%, n=90) ${ }^{14}$. Finalmente, predomina la reacción 'seguir' (el 58,3\%, $\mathrm{n}=416)$, seguido de 'afirmativo' $(28,1 \%, \mathrm{n}=200)$ y solo un $11,6 \%(\mathrm{n}=83)$ abarca las reacciones de tipo negativo. Catorce casos son ambiguos.

En suma, globalmente huevón no se reduce a actos expresivos, de meta comunicativa no compartida, de amenaza y con una reacción sentida, defensiva u ofensiva, como se supondría de un término usado como insulto o con una connotación negativa.

\footnotetext{
${ }^{14}$ Cuatro casos son ambiguos: no está claro a partir del contexto si el hablante pretende hacer una observación neutra, crítica o humorística.
} 
Ahora, al correlacionar estos cuatro parámetros más el significado semántico-pragmático se averigua en qué contextos pragmático-interaccionales huevón prevalece. Las cinco combinaciones más frecuentes, con el número de usos en cifras absolutas y porcentuales, son:

1. meta compartida - asertivo - mantener - seguir - atención

$112 \quad 15,7 \%$

2. meta compartida - asertivo - mantener - afirmativo - atención

$60 \quad 8,4 \%$

3. meta compartida - expresivo - mantener - seguir - atención

$41 \quad 5,7 \%$

4. meta compartida - expresivo - valorar - seguir - atención

28

$3,9 \%$

5. meta compartida - expresivo - mantener - afirmativo - atención

22

$3,1 \%$

Estas cinco combinaciones cubren el 36,8\% de todos los usos. Se puede concluir que huevón notablemente predomina en contextos poco comprometidos. Por el otro lado, los 16 casos de huevón de significado 'tonto' o 'comportamiento desaprobado' son de meta comunicativa no compartida, amenazantes y expresados sobre todo en actos expresivos y directivos. Sin embargo, sólo cinco de estos 16 usos provocan una reacción negativa por parte del oyente. El fragmento (11) presenta un uso de un tono un tanto irritado pero con una reacción no negativa por parte del interlocutor (i.e. la conversación sigue sin reacción verbal marcada de éste).

(11) (padre: Gen4.M, hijos: Gen3.M; padre y hijo 1 están conversando de la segunda guerra mundial)

\begin{tabular}{|c|c|c|}
\hline $\begin{array}{l}\text { Hijo } 1 \\
\text { Padre } \\
\text { Hijo } 2\end{array}$ & $\begin{array}{l}\text { Aah, pero eso siempre pasa en cualquier or } \\
{[\ldots]}\end{array}$ & $\begin{array}{l}\text { ganización imperialista poh. } \\
\text { (hacia grabadora) Quinta guerra mundial, }\end{array}$ \\
\hline $\begin{array}{l}\text { Hijo } 1 \\
\text { Padre } \\
\text { Hijo } 2\end{array}$ & Uruguay contra Ecuador. & $\begin{array}{c}\text { No,-. } \\
\text { No, eso no-. ¿Qué sé yo? Porque si yo estoy }\end{array}$ \\
\hline Padre & peleando con- si ellos estaban peleando los aleman & s con los norteamericanos. [...] \\
\hline
\end{tabular}

En suma, huevón a veces puede expresar 'tonto' en un acto expresivo y amenazante, pero su efecto ofensivo suele ser ligero.

Aún más, varios casos del corpus muestran que huevón se expresa precisamente en interacciones de tema/tono marcadamente positivos, como en (12), donde el hablante relata muy entusiasmado a sus primos sobre el precio barato de jugar en una cancha de fútbol en Bolivia y Tacna (Perú): 
(12) (primo 1: Gen3.M, primos 2 y 3: Gen2.M)

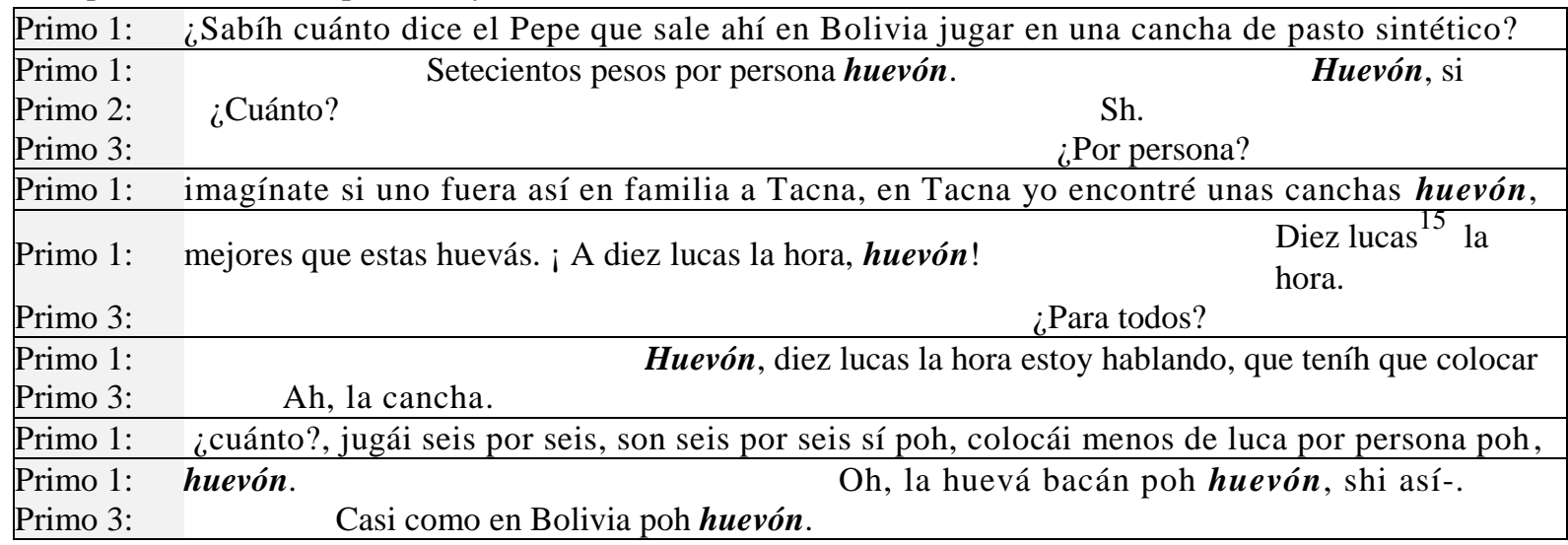

Estos usos de huevón tienen funciones fáticas: provocan un tono alegre que señala la sorpresa a una situación o algo dicho por otro; por consiguiente, consolidan las relaciones sociales entre los interlocutores (supra §2.2, Martín Zorraquino y Portolés, 1999: 4174).

Finalmente, algunos usos de huevón ocurren en situaciones en las que no se da por sentada la solidaridad entre los interlocutores, ya sea porque se están disputando (13), ya sea porque son desconocidos y/o uno es jerárquicamente superior (14).

(13) (reunión social, Gen4.M)

\begin{tabular}{|c|c|}
\hline $\begin{array}{l}\text { Miembro } 1 \\
\text { Presidente }\end{array}$ & ¿Ah? Explicándole que si nos puede vender-, que el compadre \\
\hline $\begin{array}{l}\text { Miembro } 1 \\
\text { Presidente } \\
\text { Miembro } 2\end{array}$ & diga, “Ah ya, ¿cuántos queríh”, “Tantos”, "Ya”. \\
\hline \begin{tabular}{|l|} 
Presidente \\
Miembro 1 \\
\end{tabular} & $\begin{array}{l}\text { Uno, un juego poh huevón. Si nos vende micrófonos- } \\
\qquad \text { ¡A lo sumo dos poh huevón. }\end{array}$ \\
\hline $\begin{array}{l}\text { Presidente } \\
\text { Miembro } 3\end{array}$ & "Dos, dos juegos", le ponemos, dos juegos de micrófonos. \\
\hline
\end{tabular}

El fragmento (13) muestra dos instancias de huevón en el mismo turno, uno por quien hace una observación y otro por quien reacciona a ella. Ambos casos reflejan la función atenuadora en enunciados directivos (supra §2.2, Martín Zorraquino y Portolés, 1999: 4173-4174): pretenden evitar cualquier sensación de resentimiento para mantener el equilibrio relacional entre los interlocutores. La construcción final de enunciado "poh huevón" aparece lograr este equilibrio dado que llegan a un acuerdo sin que se produzca mayor tensión.

\footnotetext{
${ }^{15}$ Luca: f. 'Mil pesos (unidades monetarias)' (Academia Chilena de la Lengua, 2010)
} 
En (14), un cliente de 30-35 años se dirige al camarero que está preparando sus tragos en la barra de un $\operatorname{bar}^{16}$.

(14)Hacia camarero: Te falta un picahielos huevón. (Gen3.M)

El cliente imprime un tono amistoso para crear solidaridad no preexistente mientras espera para llevarse sus tragos (supra §2.2, Martín Zorraquino y Portolés, 1999: 4173). La meta comunicativa de entablar amistad o expresar simpatía mediante huevón es conveniente por la atmósfera informal creada por el espacio informal del bar y el sexo masculino de ambos interlocutores ${ }^{17}$. Compárense este uso con el uso en (15), de un cliente de un negocio nocturno, que, probablemente borracho, intenta en vano de comprar algo a crédito.

(15) (dependiente 1: M, dependiente 2: F, cliente: M, edades desconocidas)

\begin{tabular}{|c|c|c|}
\hline $\begin{array}{l}\text { Dependiente } 1 \\
\text { Cliente } \\
\text { Dependiente } 2\end{array}$ & ¿Qué? Pero ¿qué queríh? ¿Crédito? & Sí, (ininteligible). \\
\hline $\begin{array}{l}\text { Dependiente } 2 \\
\text { Cliente }\end{array}$ & Sí, el lunes, seguro seguro seguro. & $\begin{array}{l}\text { No hijo, } \\
\text { (ininteligible) }\end{array}$ \\
\hline $\begin{array}{l}\text { Dependiente } 2 \\
\text { Cliente }\end{array}$ & \multicolumn{2}{|r|}{ Once mil no más, o o doce mil no más. } \\
\hline $\begin{array}{l}\text { Dependiente } 2 \\
\text { Cliente }\end{array}$ & Está muy alto. & Después que pague eso. \\
\hline $\begin{array}{l}\text { Dependiente } 2 \\
\text { Cliente }\end{array}$ & $\begin{array}{l}\text { Después que pague eso. } \\
\text { ¿De qué? }\end{array}$ & ¿De qué (ininteligible)? \\
\hline $\begin{array}{l}\text { Dependiente } 2 \\
\text { Cliente }\end{array}$ & Después que pague eso. & Pago el lunes seguro huevón. \\
\hline $\begin{array}{l}\text { Dependiente } 2 \\
\text { Cliente }\end{array}$ & Por favor. & lemos. \\
\hline
\end{tabular}

Aquí, huevón también crea solidaridad y complicidad además de atenuar el acto de habla directivo: el cliente quiere que los dependientes depositen su confianza en él para que le den el crédito. Sin embargo, la situación es bastante más formal: no se encuentran en un bar sino en un negocio, la actividad de compra y venta es principalmente transaccional y el interlocutor es una mujer que además parece de edad mayor que el cliente (ella le trata de "hijo" en su segundo turno). Además, la dependiente le trata de usted en "después que pague eso", con lo que señala

\footnotetext{
${ }^{16}$ Dado el ruido en el bar, la grabadora no registra una posible respuesta del camarero. Por lo tanto, no se sabe cómo éste reacciona a la observación del cliente.

17 Notamos que esta relación no corresponde al prototipo de ninguno de los seis ámbitos seleccionados. La interacción resulta de la naturalidad de las grabaciones: se registró durante la actividad de tres amigos que están saliendo juntos y que en un momento están en un bar. Estos también compran algo en un negocio nocturno más adelante, de lo que resulta el fragmento (17).
} 
querer mantener distancia. Finalmente, ella ya ha dejado claro varias veces que no va a dar el crédito. Por lo tanto, el uso del huevón solidario e informal da una impresión desubicada, maleducada, inesperada y por lo tanto descortés. El estado de embriaguez del cliente puede explicar esta actitud inoportuna.

En conclusión, huevón se usa en contextos muy similares del MDD hombre del español peninsular: predomina su uso fático con el significado 'atención' pretendiendo ocasionar un tono alegre, reforzar las relaciones sociales, atenuar expresiones directivas o disconformes y crear solidaridad. Su uso apenas provoca una reacción negativa del oyente. Por consiguiente, la mayoría de los usos son políticos, es decir apropiados y no marcados. Se observan estas mismas funciones de atenuación y de vinculación y creación de solidaridad para güey (Kleinknecht, 2013: 154, 158), dude (Kiesling, 2004: 291) y mate (Rendle-Short, 2009: 262).

\section{Conclusión y perspectivas futuras}

Este artículo ha examinado el uso de la FdT nominal huevón en el español de Chile esbozando un perfil de sus características sociopragmáticas más destacadas. Se ha comprobado que el uso más común de huevón es como MDD con la función de atenuar actos de habla potencialmente amenazadores y de consolidar o crear la solidaridad entre interlocutores. Su uso es considerado apropiado en contextos muy informales. La informalidad se deduce del espacio físico, la relación de proximidad existencial íntima entre los interlocutores, la finalidad comunicativa interpersonal, la jerarquía igualitaria entre los interlocutores por su edad, sus roles funcionales y la solidaridad de ser del mismo sexo (masculino). Cuando el contexto relacional y situacional facilitan el uso de huevón, lo intercambian frecuentemente no solo jóvenes sino personas/hombres de todas las edades, con excepción de niños que lo emplean menos. Fuera del contexto apropiado, se evita generalmente el término.

Aun así, quedan pendientes varias cuestiones. Primero, dada la refutación de vincular el uso de huevón al habla de jóvenes, es útil reafirmar el uso de huevón y otros MDD de este tipo en corpus más amplios, que incluyen no solo todas las generaciones, sino también personas adultas y mayores en todo tipo de roles funcionales. Hasta ahora, pocos estudios se han centrado en interacciones muy informales, íntimas y no-institucionales entre adultos y/o mayores. 
Segundo, varios resultados han dejado claro que huevón es principalmente un símbolo de identidad colectiva de hombres y que su estigma o connotación negativa persiste más en el habla y en la percepción de mujeres. Además, los estudios de dude (Kiesling, 2004), hombre y home (Cuenca y Torres, 2008), mate (Rendle-Short, 2009) y guëy (Kleinknecht, 2013) muestran tendencias muy parecidas. Esta recurrencia hace surgir varias preguntas. ¿Por qué se forman estos términos (masculinos) y con la misma función de reforzar la solidaridad e identidad colectiva en interacciones entre hombres, mientras que solo aparecen de manera limitada en el habla de mujeres? Dada la gran relevancia interaccional de sus funciones fáticas, ¿sería que las mujeres apelan más a otros recursos (no-)verbales para expresar una identidad colectiva interna? Tercero, queda pendiente el examen profundo de las demás derivaciones de la familia léxica de hueva. Por ejemplo, ¿cuáles son las funciones y rasgos sociopragmáticos de la FdT huevona? y, ¿en qué medida cada una de las derivaciones adjetivales, sustantivas y verbales mantienen un valor negativo por su origen semántico-pragmático despectivo y vulgar?

Finalmente, varios usos de huevón han resultado ambiguos a la hora de determinar su valor semántico-pragmático exacto. Además de su posición sintáctica y realización fonética, se podría examinar la validez de rasgos prosódicos como la duración de las sílabas (ritmo), la sonoridad (acento) y la altura del sonido (entonación) para desambiguar la interpretación de huevón. Con todo, sería interesante verificar en qué medida estos parámetros apuntan hacia un proceso de gramaticalización de huevón.

\section{Bibliografía citada}

ACADEMIA CHILENA DE LA LENGUA, 2010: Diccionario de uso del español de Chile, Santiago: MN Editorial Ltda.

ALBELDA, Marta, 2008: "Atenuantes en Chile y España: distancia o acercamiento" en Antonio BRIZ y otros (eds.): Cortesía y conversación: de lo escrito a lo oral. IIIr Coloquio Internacional de EDICE, Valencia/Estocolmo: Universidad de Valencia/Edice, 98-113.

BRIZ, Antonio, 2004: "Cortesía verbal codificada y cortesía verbal interpretada en la conversación” en Diana BRAVO y Antonio BRIZ (eds.): Pragmática sociocultural: estudios sobre el discurso de cortesía en español, Barcelona: Ariel, 67-93. 
_ 2005: "Eficacia, imagen social e imagen de cortesía. Naturaleza de la estrategia atenuadora en la conversación cotidiana española" en Diana BRAVO (ed.): Estudios de la (des)cortesía en español. Categorías conceptuales y aplicaciones a corpora orales y escritos, Estocolmo/ Buenos Aires: Edice/Dunken, 53-91.

CORTÉS, Saide, 2009: "Expresiones zoonímicas en el habla popular chilena", Boletín de Filología 44 (2): 243 - 261.

CUENCA, María Josep y Marta TORRES VILATARSAN, 2008: "Usos de hombre/home y mujer/dona como marcadores del discurso en la conversación coloquial”, Verba 35: 235-256.

FORUM.WORDREFERENCE, 2005: "Huevon, Guewon, gueon", [http://forum.wordreference. com/showthread.php?t=51385\&langid=13, fecha de consulta: 16 de enero de 2013]

HELINCKS, Kris, 2012: "La variación social y estilística del voseo chileno en diferentes géneros televisivos”, Revista internacional de lingüística iberorrománica 19, 185-211.

—, (en preparación): "Corpus de conversaciones espontáneas del español chileno en Iquique", banco de datos no publicado. Gent: Universiteit Gent.

HERNES, Solfrid, 2011: El uso de palabras tabúes en el lenguaje juvenil de Santiago de Chile y Oslo. Un estudio contrastivo. Tesis de maestría, Universidad de Bergen.

JØRGENSEN, Annette Myre, 2011: "Formas de tratamiento: Los vocativos en el lenguaje juvenil de Madrid, Buenos Aires y Santiago de Chile" en Leticia COUTU y Célia LOPES (eds.): Las Formas de Tratamiento en español y en portugués. Variación, Cambio y Funciones conversacionales, Niterói: Editora da UFF, 125-148.

KIESLING, Scott, 2004: “Dude”, American Speech 79 (3): 281-305.

KLEINKNECHT, Friederike, 2013: "Mexican güey - from vocative to discourse marker: a case of grammaticalization?" en Barbara SONNENHAUSER y Patrica Noel Aziz HANNA (eds.): Vocative! Addressing between system and performance, Berlin: De Gruyter Mouton, 235-268.

MARTÍN ZORRAQUINO, María Antonia y José Portolés LÁZARO, 1999: "Los marcadores del discurso" en Ignacio BOSQUE y Violeta DEMONTE (dirs.): Gramática descriptiva de la lengua española. Entre la oración y el discurso; Morfología, Madrid: Espasa, 4051-4123.

PORTOCARRERO, Cosme, 1998: La palabra huevón. Santiago de Chile: Lom Ediciones.

RENDLE-SHORT, Johanna, 2009: "The Address Term Mate in Australian English: Is it Still a Masculine Term?", Australian Journal of Linguistics 29: 2, 245-268. 
ROJAS, Darío, 2012: “Huevón como MDD en el español de Chile: huellas de un proceso de gramaticalización", Revista de humanidades 25: 145-164.

SÁEZ-GODOY, Leopoldo (ed.), 1983: "Una familia léxica del español común e informal de Chile: hueva y sus derivados" en Estudios lingüísticos en memoria de Gastón CarrilloHerrera. Bonn: Romanistischer Verlag, 133-152.

TORREJÓN, Alfredo, 1986: “Acerca del voseo culto de Chile”, Hispania 69 (3): 677-683. 